\title{
Current Treatment of Medulloblastoma
}

\section{Medulloblastoma ve Güncel Tedavi Yaklaşımları}

\author{
Özlem Derinalp Or ${ }^{1}$, Yıldız Yükselen Güney ${ }^{3}$, Ayşen Dizman ${ }^{2}$, Gonca Altınışık İnan² ${ }^{2}$, Gülhan Güler \\ Avci $^{2}$,Muzaffer Bedri Altundag ${ }^{2}$ \\ ${ }^{1}$ Adana Numune Eğitim Ve Araştırma Hastanesi, Radyasyon Onkolojisi Kliniği, Adana, Türkiye \\ ${ }^{2}$ Dr. A. Y. Ankara Onkoloji Eğitim Ve Araştırma Hastanesi, Radyasyon Onkolojisi Kliniği, Ankara, Türkiye \\ ${ }^{3}$ Fırat Üniversitesi Tıp Fakültesi, Radyasyon Onkolojisi Ana Bilim Dalı, Elazı ̆̆, Türkiye
}

\section{ÖZET}

Medulloblastom çocukluk çağının ikinci en sık görülen ve en malign beyin tümörüdür. Yetişkinlerde ise oldukça nadir rastlanır. Medulloblastom tanılı hastalar tedavi öncesi standart risk ve yüksek riskli olmak üzere iki gruba ayrılır. Cerrahi rezeksiyonu takiben kraniospinal radyoterapi tedavinin temelini oluşturur. Günümüzde tedaviye kemoterapinin eklenmesi sağkalımın artmasına katkıda bulunmuştur. Ayrıca kemoterapinin eklenmesi ile total radyoterapi dozu da düşürülmüştür. Tedavide yeni kemoterapik ajanların geliştirilmesi ve yeni radyoterapi teknikleri hastaların uyumunu arttırmıştır.

Anahtar Kelimeler: Medulloblastom; Kraniospinal 1şınlama; Radyoterapi

\begin{abstract}
Medulloblastoma is the second most common and most malignant brain tumor in children. But in adults medulloblastomas occur rarely. The patients with medulloblastoma are stratified two groups before treatment: avarage risk and high risk. Surgical resection followed by craniospinal irradiation has been the mainstay of medulloblastoma therapy. In recent years, chemotherapy has been introduced to reduce the total dose of radiation and increase the survival rate. The new radiotherapy techniques and chemotherapy agents increase compliance to treatment.
\end{abstract}

Key words: Medulloblastoma; Craniospinal radiotherapy; Radiotherapy

\section{Genel Bilgiler}

Medulloblastom santral sinir sisteminin (SSS) primitif nöroektodermal tümörüdür. Serebelluman köken alır, 4. ventriküle dek uzanır $(1,2)$. Çocukluk çağı beyin tümörlerinin \%2030 'unu ve tüm posterior fossa (PF) tümörlerinin \%40'1nı oluşturur ve 5-10 yaşında pik yapar (3). Yetişkinlerde ise bu oran $\% 1$ kadardır. Yetişkinde görüldüğü yaş aralı̆ğ 20 34 ve 55-64 yaş arasıdır. Erkeklerde iki kat daha siktır (4).

Ekstranöral tutulum kemik, kemik iliği, lenf nodu, karaciğer ve akciğerde olmakla beraber nadirdir ve genellikle hastalığın son dönemi ile ilişkilidir. Relapsların en sık görüldügü yer hem yetişkin hem çocuklarda PF'dir. Buna rağmen geç relapslar yetişkinlerde daha sıktır. Yetişkinlerde relapsa kadar geçen süre ortalama 26 ay olup \%29'u tedaviden sonraki 5 yll ya da daha uzun bir sürede ortaya çıkar (5).

\section{Klinik Bulgular}

Klinik bulgular non-spesifik olup intrakranial basınç artışı ve hidrosefaliye bağlı gelişir. Başağrısı, bulantı ve kusma tipik bulgulardır. Kafa çifti tutulumuna bağlı bulgular, papil ödem, irritabilite, letarji ve nörolojik defisit eşlik edebilir $(6,7)$. Bazı hastalarda sırt ağrısı, hemiparezi, görme alanı defekti, denge bozukluğu, işitme kaybı ve nöbet diğer eşlik eden semptomlardır $(8,9)$. Kraniyal sinir paralizisi intrakraniyal basınç artışına ya da doğrudan tümör basısına bağlı olarak ortaya çıkabilir (\%21). En sik IV. ve VI. sinir paralizisine rastlanır (9).

\section{Tanı}

Tanı ilk olarak anamnez, fizik muayene ve laborotuvar testleri ile başlar. Tanıda manyetik rezonans görüntüleme (MRG) standarttır. MRG'de kitle T1'de hipointens, T2'de hiperintens görünür (Resim 1). Güçlü kontrast 


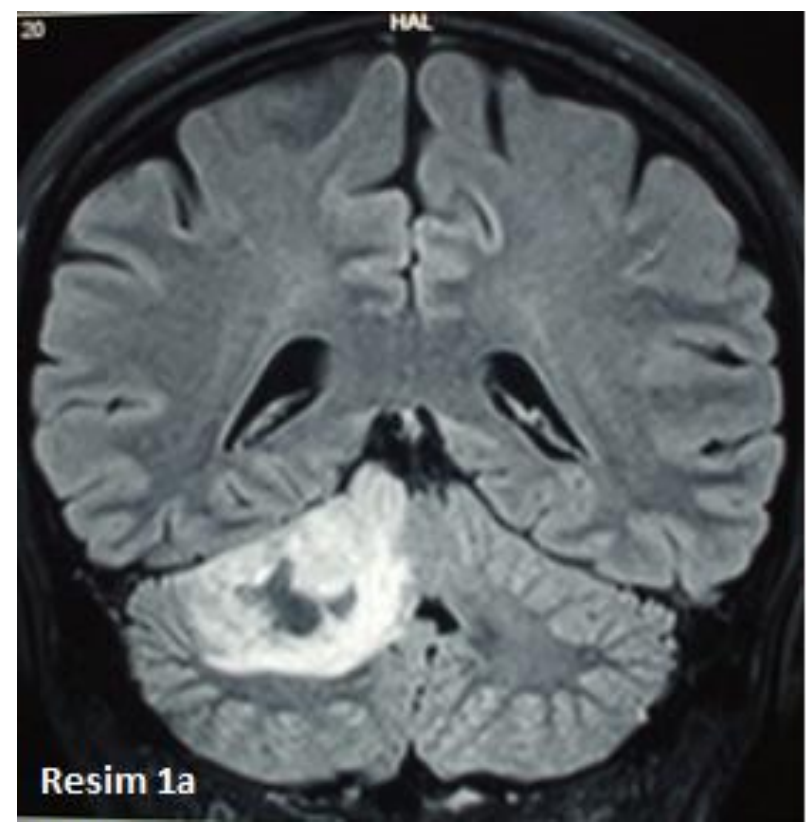

Resim 1: Medulloblastom MRG görüntüsü

tutulumu saptanır. Nekroz, kanama ve kist formasyonu tümörün karakteristik bulgularıdır. Cerrahi öncesi beyin ve spinal MRG evreleme ve tedavi alanını belirleme açısından gereklidir. Cerrahi sonrasında ise risk grubu için rezidü tümörü değerlendirmek amaciyla ilk 24-48 saat içinde beyin MRG çekilmelidir. Hastaların \%30-40'ında başvuru sırasında beyin-omurilik sıvısı (BOS) tutulumu ve spinal tutulum vardır. Leptomeningeal tutulumu saptamak ya da ekarte etmek için spinal MRG gerekir (10). Beyin-omurilik sıvisı tutulumunu saptamak için lomber ponksiyon yapılmalıdır. Medulloblastom kemik ve kemik iliğine sik metastaz yapar. $\mathrm{Bu}$ metastazlar tüm vücut kemik sintigrafisi ve kemik iliği biyopsisi ile saptanabilir. Kesin tanı cerrahi sonrası histopatolojik inceleme ile konur (9).

\section{Evreleme}

Medulloblastom için en sık kullanılan evreleme sistemi 1969'da tanımlanan Chang evreleme sistemidir. $\mathrm{Bu}$ sistemde tümör

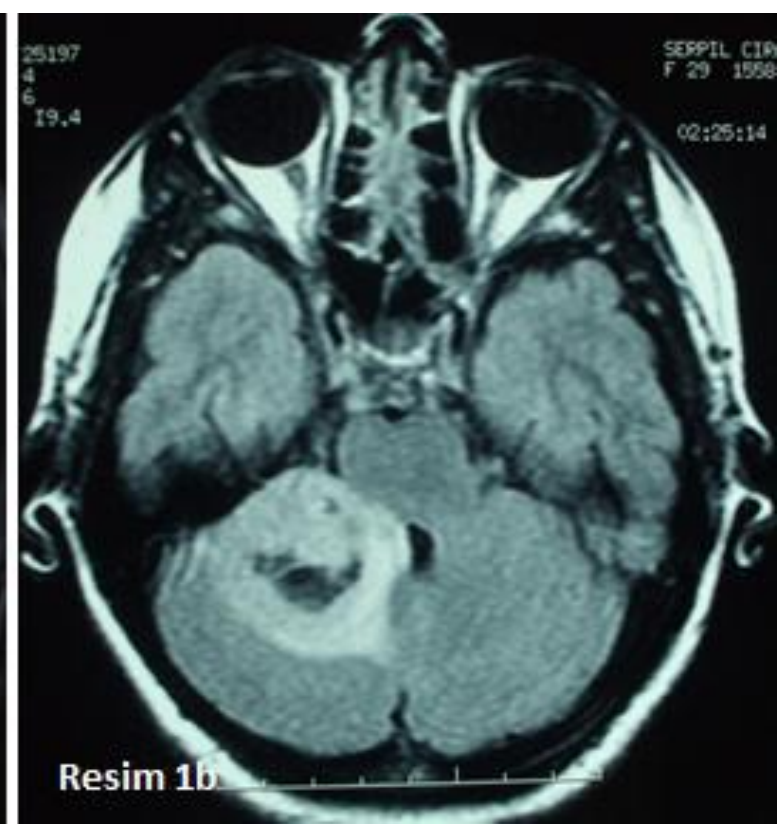

büyüklüğü ve görüntüleme yönteminden yararlanılır (11). Ancak tanı ve tedavi yöntemlerindeki gelişmeler ve tümörün biyolojik özelliklerinin daha fazla anlaşılmasıyla birlikte evreleme dışında prognostik faktörler belirlenmistir (9). Son yıllardaki çalışmalar $\mathrm{T}$ evresinin prognozda yeri olmadığını göstermiş, yaş ve metastaz varlı̆g ile prognoz arasındaki ilişki vurgulanmıştır $(9,11)$.

\section{Risk Sinıflandırması ve Prognostik Faktörler}

Medulloblastomda tedavinin en önemli kısmı prognostik faktörlerin belirlenmesidir. Tedaviden önce prognostik faktörler göz önüne alınarak hastalara uygun tedavi modaliteleri seçilir. Hastalar tedavi öncesi standart risk ve yüksek risk olmak üzere iki risk grubuna ayrilır (Tablo 1). Cerrahi sonrası radyoterapi dozları ve kemoterapi ajanları bu risk gruplarına göre belirlenir (Tablo 1) (12-14).

Tablo 1: Risk grupları

\begin{tabular}{|l|l|l|}
\hline & Standart risk & Yüksek risk \\
\hline Yaş & $>3$ & $\leq 3$ \\
\hline Metastaz $(M)$ & Yok $(\mathrm{M} 0)$ & Var $(\mathrm{M}+)$ \\
\hline Postoperatif kalıntı & $<1.5 \mathrm{~cm}^{3}$ & $\geq 1.5 \mathrm{~cm}^{3}$ \\
\hline
\end{tabular}

Çocukluk çağında erkek cinsiyet kötü prognostik faktördür (11). Küçük çocuklarda metastatik hastalık riski daha yüksektir. Bunun nedeni de bu çocuklarda daha çok subtotal rezeksiyon uygulanmasi ve yan etkiler nedeniyle konvansiyonel dozlarda kraniospinal 
radyoterapi (KSRT) uygulanamamasıdır. Tümör biyolojisi de daha agresif özellikler göstermektedir (9). Children's Oncology Group (COG) tarafindan yürütülen bir çalısmada hastalıksız sağkalım oranı 3 yaş altı olgularda $\% 32$ iken, 3 yaş üstü olgularda $\% 58$ bulunmuştur (15).

Medulloblastom tan1lı hastalarda total ya da totale yakın rezeksiyon önerilmektedir. Subtotal rezeksiyonun kötü prognozla ilişkili olduğu çalışmalarla gösterilmiştir. Postoperatif kalıntı tümör varlığının $\left(\geq 1.5 \quad \mathrm{~cm}^{3}\right) \quad \mathrm{M} 0$ hastalarda dahil prognostik olarak anlamlı olduğu birçok çalışmada ortaya konmuştur (15). Metastatik evre en önemli prognoz göstergesi olarak kabul edilmektedir (1). COG921 çalışmasında 5 yıllık genel sağkalım M0 ve M1 hastalarda \%78 iken, M2 ve M3 hastalarda \%21 olarak saptanmıştır (15).

\section{Tedavi Yaklaşımı}

Medulloblostom için günümüzde kabul gören tedavi şeması 3 yaşın üzerindeki çocuklarda maksimum cerrahi rezeksiyon, KSRT ve kemoterapidir $(11,16)$. Üç yaş altındaki hastalarda radyoterapi (RT) uygulaması beyin gelişimi henüz tamamlanmadığ 1 için 3 yaş sonrasina geciktirilir. $\mathrm{Bu}$ sürede hastalara yoğun kemoterapi uygulanmaktadır (10).

\section{Cerrahi Tedavi}

Medulloblastom tedavisinin ilk ve en önemli basamağ cerrahi tedavi olup tümör dokusunun maksimum çıkarılması ve kalıntı kalmaması hedeflenir. Postoperatif kalıntı miktarı en önemli prognostik faktörlerdendir (12-14). Hidrosefali ve kafa içi basınç artışının eşlik ettiği hastalarda cerrahi öncesi yüksek doz deksametazon tedavisi başlanmalı, gerekirse ventrikuloperitoneal şant takılmalıdır (15).

\section{Kemoterapi}

Medulloblastom kemoterapiye hassas tümörlerdendir. Kemoterapinin medulloblastom hastalarında yarar sağladığı ilk kez International Society of Pediatric Oncology (SIOP) tarafindan gösterilmiş, ardından Chidren's Cancer Group (CCG) ve Pediatric Oncology Group (POG) çalışmaları ile de desteklenmiştir. Kemoterapi hastalık kontrolü- nü ve sağ kalımı arttırır, metastatik hastalıkta tedavi avantajı sağlar (17).

Standart riskli medulloblastom hastalarında cerrahi sonrası kemoterapi ve düşük doz KSRT (23.4 Gy) ile birlikte PF'ya klasik doz radyoterapi $(55.8 \mathrm{~Gy})$ uygulamasının yüksek genel sağkalım oranı sağladığı gösterilmiştir (18-20). Packer ve ark.'nın yaptığı çalışmada postoperatif RT ile birlikte vinkristin ve RT ardindan adjuvan kemoterapi (vinkristin, lomustin ve sisplatin kombinasyonu) verilmiș (11). Bu rejim günümüzde altın standart olarak kabul edilmektedir. Ancak erken toksisite nedeniyle doz ayarlaması gerektirmiş, özellikle ototoksisite saptanmıştır (21). Kemoterapi KSRT dozlarının azaltılmasına olanak sağlamış ve SSS dişında metastatik odakların gelişmesi riskini azaltııştır (18-22).

Yüksek riskli hastalarda yapılan iki randomize çalışmada adjuvan kemoterapinin yararı gösterilmiştir $(17,18)$. POG çalışmasında RT ve kemoterapi alan yüksek riskli grupta sadece RT alan gruba kıyasla daha iyi sağkalım oranları görülmüştür. Genel sağkalım avantajının erkeklerde ve 5 yaş ve üzerindeki hastalarda en fazla olması dikkat çekmektedir (\%82 vs \%50.1) (17). SIOP II çalışması ile yüksek riskli hastalarda RT ve adjuvan kemoterapinin belirgin genel sağkalım avantajı sağladığı gösterilmiştir (18).

\section{Radyoterapi}

Medulloblastom radyosensitif bir tümör olup cerrahi sonras1 adjuvan KSRT uygulamas1 3 yaş üzerindeki çocuklarda standarttır. Ancak tedaviye bağlı, özellikle çocuklarda uzun dönem kognitif, nöroendokrin ve nöropsikolojik bozukluklar ve sekonder malignite gelişme riski yüksektir $(9,14)$. Bu nedenle doz azaltılmasına yönelik çalışmalar yapılmaktadır. Standart risk hastalarda eş zamanlı kemoterapi ile KSRT'de doz azaltılmasını gösteren (KSRT 23.4 Gy, PF 55.8 Gy) CCG 9892 çalışmasında hastalıksız sağkalımda bir düşüş izlenmemiştir (3 ve 5 y1ll1k, \%86 ve \%79). Günümüzde bu protokol standart risk grubu için kabul görmüştür (11) (Tablo 2). POG 8631/ CCG923 çalışmasında kemoterapi olmadan doz azaltılmaya çalışıldığında (KSRT 23.4 Gy x 36 Gy, PF toplam 54 Gy) ise hem GS hem DFS olumsuz etkilenmiştir (23).

Çalışmalar sonucunda RT dozunun azaltılıp tedaviye kemoterapi eklenmesinin sağkalım 
üzerine olumsuz etkisi olmadığı gösterilmiştir.

başarılı sonuçlar elde edilmiştir (Tablo 2) Aksine tedaviye uyum ve devam açisından $(9,11,22)$.

Tablo 2: Tedavi Șemas1

\begin{tabular}{|l|l|l|}
\hline & Çocukluk Çă̆ & Yetişkin \\
\hline Standart Risk & KSRT:23.4 Gy & KSRT:30-36 Gy VEYA \\
& PF:54 Gy (eşzamanlı vinkristin) & $\begin{array}{l}\text { 23.4 Gy +KT (ȩ̧zamanl1) } \\
\text { PF:55.8 Gy }\end{array}$ \\
\hline Yüksek Risk & KSRT:36-39 Gy & KSRT:36 Gy \\
& PF:54 Gy (eşzamanlı vinkristin) & PF:55.8 Gy \\
\hline
\end{tabular}

Günümüzde gelișen teknolojinin sonucu olarak RT cihazları ve uygulama tekniklerindeki gelişmeler nedeniyle radyoterapiye bağlı yan etkiler de azalmaktadır. Yoğunluk ayarlı RT
(YART) ile tümöre yüksek doz verilirken, kritik yapılar en az doz almakta, doz dağılımında, iyileşme sağlanmaktadır (Şekil 1) (24).

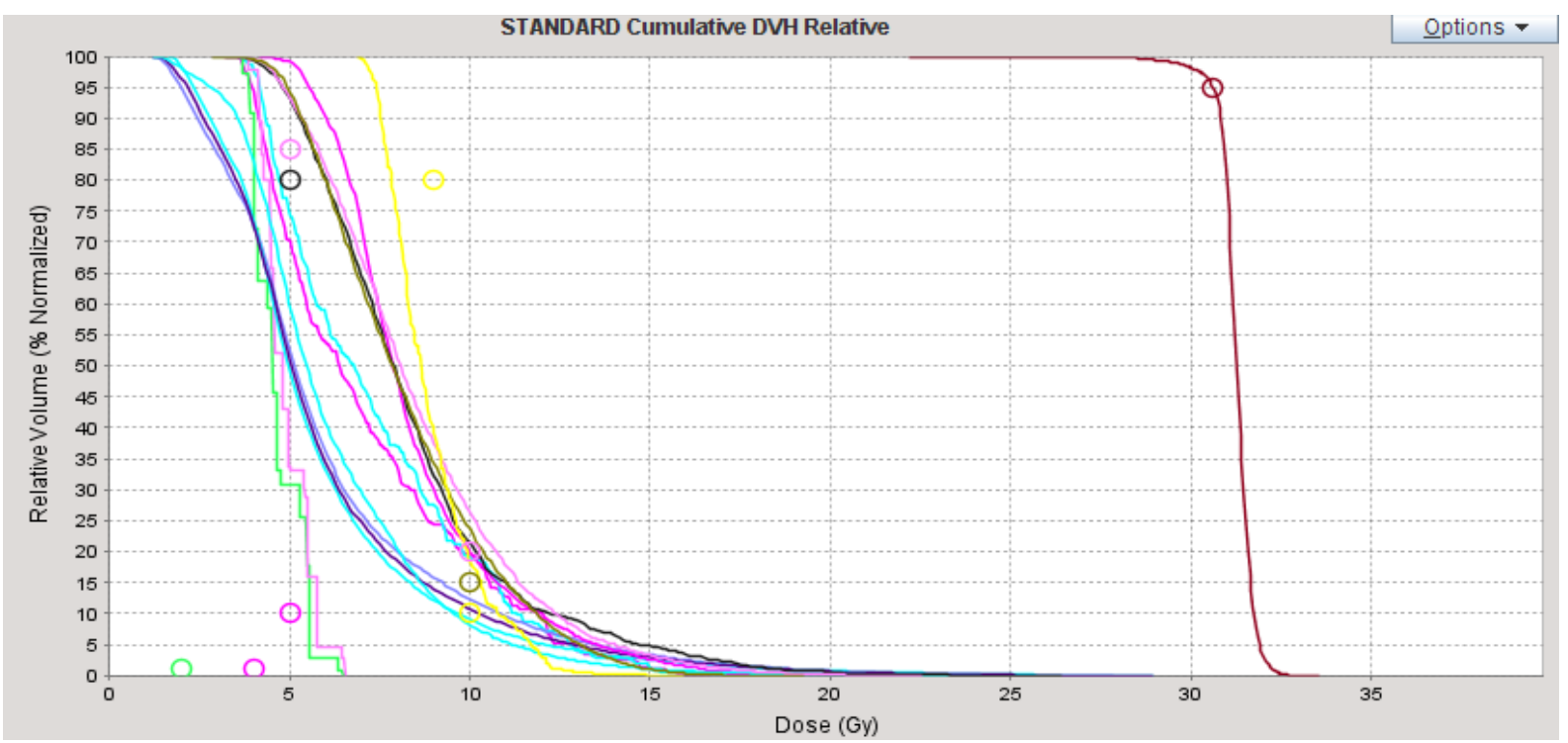

Şekil 1: Helikal Tomoterapi (Hi·Art® ) ile planlama: Doz-Volüm Histogramı (Dr. A.Y. Ankara Onkoloji Eğitim ve Araştırma Hastanesi)

Diğer taraftan, sahip olduğu dozimetrik avantajlar nedeniyle (hedef volüm dışında hızlı doz düşüşü) özellikle çocuklarda yaşam kalitesi ve ikincil malignite gelişmesi, nörokognitif, nöroendokrin gibi yan etkiler açısından proton RT önerilmektedir (25). Ancak yüksek maliyet nedeniyle günümüzde yaygın kullanılmamaktadır. Üç boyutlu (3B) konformal, YART ve proton RT'sini karşılaştıran bir çalışmada kritik organ dozları incelenmiștir. Kohlea, verilen dozun 3B tedavi ile $\% 90$, YART ile $\% 33$ ve proton tedavisi ile $\% 2$ 'sini alırken; hipofiz sirayla $\% 63, \% 19$ ve $\% 0$ 'ını almıştır. Diğer organlarda da sonuçlar benzerdir (26).

\section{Radyoterapi Tekniği}

Kraniyospinal radyoterapide hedef volüm; tüm beyin, spinal kord ve tüm meninkslerden oluşmaktadır. İki boyutlu (2B) konvansiyonel RT yönteminde hasta prone pozisyonunda yatar ve bu pozisyonda termoplastik maskeler yardımı ile immobilizasyon sağlanır $(31,32)$. Tüm beyin iki lateral alandan, spinal kord ve meninksler ise posterior spinal alandan tedavi edillir. Lateral alanların alt sınırı ile posterior alanın üst sınırı arasında doz çakışmasına engel olacak şekilde bir mesafe (gap) bırakılır. Bu alanda oluşabilecek doz düşüşü ya da doz artışına engel olmak için, bu gap mesafesi tedavi boyunca belli aralılarla kaydırılır. Spinal alanın alt sınırı S2'nin altından geçecek şekilde belirlenir (Resim 2) (10,11). Modern teknolojik cihazlarla uygulanan tekniklerde 
(3B konformal, YART, Tomoterapi vb) hedefe yönelik daha homojen doz dağılımları sağlanırken, hedef dışı organlar en az dozu almaktadır (Tablo 3) (27). Bu tekniklerde hasta supin pozisyonunda yatarak tedavi edilebilmektedir (Resim 3). Bu pozisyon hem hasta için konfor sağlarken diğer taraftan, anestezi gereken durumlarda hava yolunun daha iyi kontrol edilmesine imkan vermektedir (28-30).

$\mathrm{Bu}$ yöntemlerde hedef volüm, hastanın tedavi pozisyonunda çekilen bilgisayarlı tomografi (BT) görüntüleri üzerinden belirlenir (29-31). MRG spinal volümün alt sınırının daha güvenli işaretlenmesini sağlar. T2 ağırlıklı MRG'de tekal kesenin sonlandığı yerden geçilebilir Spinal alan için; hasta hareketi, setup hataları gözönüne alınarak klinik hedef volüme (CTV) her yönden $5 \mathrm{~mm}$ sinır verilerek planlanan hedef volüm (PTV) oluşturulur (31- 32). Bu planlama yöntemleri ile konvansiyonel yöntemlerde yaşanan alan çakışması sorunu da ortadan kalkmaktadır.Alan birleşim yerlerindeki doz kontrolü sağlanabilmektedir (Resim 3) (Tablo 3) $(24,25)$.

Gelişen tedavi yöntemlerinden en uygunu, özellikle çocuk hastalarda proton 1şınları ile RT'dir. Proton RT ile hedefe yüksek doz verilirken hedef dışı bölgelerde doz çok düşüktür. Doz dağılımlarında ciddi iyileşmeler sağlanır. Çıkış doza neden olmadığı için özellikle pediatrik tümörlerde tedaviye bağl1 komplikasyonları azaltmaktadır

(24).
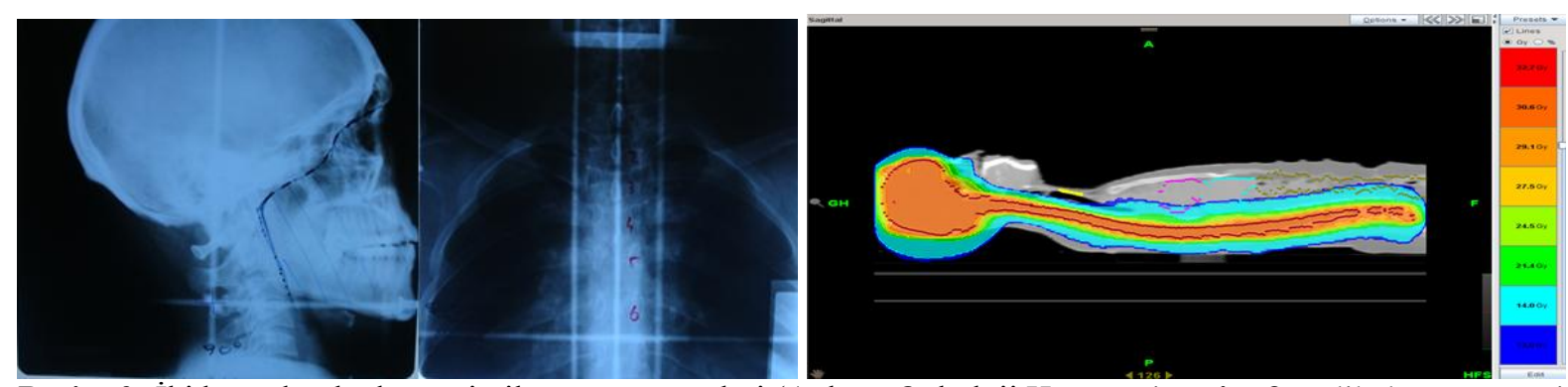

Resim 2: İki boyutlu planlama-similasyon görüntüleri (Ankara Onkoloji Hastanesi, resim 3:Helikal Tomoterapi $\left(\mathrm{Hi} \cdot \operatorname{Art}{ }^{\circledR}\right)$ ile planlama: Hasta pozisyonu (Dr. A.Y. Ankara Onkoloji Eğitim ve Araştırma Hastanesi)

Tablo 3: Tablo 3: Kritik organların 10 Gray doz aldığı volüm (\%) (33)

\begin{tabular}{|l|l|l|l|}
\hline Kritik Organ & $2 \mathrm{~B}$ & $3 \mathrm{~B}$ & YART \\
\hline Kalp & 76 & 55 & 10 \\
\hline Karaciğer & 38 & 24 & 4 \\
\hline Özefagus & 19 & 8 & 4 \\
\hline Barsak & 62 & 35 & 14 \\
\hline Sağ Böbrek & 54 & 7 & 7 \\
\hline Sol Böbrek & 43 & 4 & 6 \\
\hline Sağ Akciğer & 36 & 15 & 11 \\
\hline Sol Akciğer & 22 & 5 & 5 \\
\hline Vertebra & 100 & 98 & 99 \\
\hline Tiroid & 100 & 100 & 100 \\
\hline
\end{tabular}

\section{Kraniyospinal Radyoterapiye Bağlı Komplikasyonlar}

- Akut etkiler (ilk 6 hafta): Saç dökülmesi, radyasyon dermatiti, yorgunluk, bulant1kusma, otit (33).

- Subakut etkiler (6-12 hafta): Somnolans, kapiller geçirgenliğin artışı ve geçici demyelinizasyon (33).

- Geç etkiler (3 ay-3 yıl): Radyasyon nekrozu, lökoensefalopati, duyma kaybı, retinopati, katarakt, görme değişiklikleri, endokrin bozukluklar, nörokognitif bozukluklar, infertilite, ikincil malignite (33).

\section{İzlem}

Önerilen izlem süresi standart risk grubundaki hastalarda ilk 2 y1l 3 ayda bir beyin MRG, 6 ayda bir spinal MRG; 2-5 y1l aras1 6 ayda bir ve 5 yıldan sonra y1llik MRG takibi şeklindedir. Yüksek risk grubundaki hastalarda ise ilk 2 y1l 3 ay, sonrasinda ise 6 ay aralarla 
beyin ve spinal MRG önerilir. Semptom varlığında ise direk MRG istenmelidir (34).

\section{Sonuç}

Medulloblastom tanilı hastalarda klinik, radyolojik, patolojik ve genetik faktörler de göz önüne alınarak risk grupları belirlenmeli ve tedavi şeması uygulanmalıdır. Yetişkin medulloblastom hastalarında prospektif veriler sinırlı olup tedaviler heterojendir. RT cerrahiden sonra standart tedavi olup 1953'ten beri KSRT uygulanmaktadır. Yeni gelişmiş tedavi modaliteleri, özellikle RT cihazları ile mortalite ve morbidite oranları azalmaktadır.

\section{Çıkar İlişkisi: Yoktur}

\section{Kaynaklar}

1. Yazigi-Rivard L, Masserot C, Lachenaud J, DieboldPressac I, Aprahamian A, Avran D, Doz F. [Childhood medulloblastoma]. Arch Pediatr 2008;15:1794-804

2. Brada M. Pathology and Genetics of Tumours of the Nervous System. Br J Cancer 2001;84:148

3. Heideman RL, Packer RJ, Albright LA, Freeman CR, Rorke LB. Tumors of the central nervous system. In: Pizzo PA and Poplack DG, editors.Principles and Practice of Pediatric Oncology, 3rd edition. Philadelphia,USA: Philip, Lippincott-Raven, 1997

4. Kun LE. Brain tumors in children. In: Perez CA and Brandy LW, editors. Principles and Practice of Radiation Oncology. 3rd Edition. Philadelphia,USA: Lippincott-Raven, 1997

5. Bloom HJ: Medulloblastoma: Prognosis and prospects Int J Radiat Oncol Biol Phys 1977;2:10311033

6. Dai CY, Nakamura JL, Haas-Kogan D. Central Nervous System. In: Hansen EK, Roach III M, editors. Handbook of evidence based radiation oncology. New York, NY: Springer; 2006:15-54

7. Kun LE, Tarbell NJ. Current Treatment and Contraversies in Childhood Brain Tumors. In: ASTRO Education session. Atlanta, GA; 2004

8. Polkinghorn WR, Tarbell NJ. Medulloblastoma: tumorigenesis, currentcinical paradigm, and efforts to improve risk stratification. Nat Clin Pract Oncol 2007;5:295-304

9. Rood BR, Macdonald TJ, Packer RJ. Current treatment of medulloblastoma: Recent advances and future challenges. Semin Oncol 2004;31:666-75

10. Vezina LG, Packer RJ. Infratentorial brain tumors of childhood. Neuroimaging Clin North Am 1994;4:423-436

11. Packer RJ, Goldwein J, Nicholson HS, et al. Treatment of children with medulloblastomas with reduced-dose craniospinal radiation therapy and adjuvant chemotherapy: A Children's Cancer Group Study. J Clin Oncol 1999;17:2127-36

12. Yao MS, Mehta MP, Boyett JM, et al. The effect of $\mathrm{M}$-stage on patterns of failure in posterior fossa pri- mitive neuroectodermal tumors treated on CCG-921: a phase III study in a high-risk patient population. Int J Radiat Oncol Biol Phys 1997;38:469-76

13. Lamont JM, McManamy CS, Pearson AD, Clifford SC, Ellison DW. Combined histopathological and molecular cytogenetic stratification of medulloblastoma patients. Clin Cancer Res 2004;10:5482-93

14. Chintagumpala M, Berg S, Blaney SM. Treatment contraversies in medulloblastoma. Curr Opin Oncol 2001;13:154-9

15. Zeltzer PM, Boyett JM, Finlay JL, et al. Metastasis stage, adjuvant treatment, and residual tumor are prognostic factors for medulloblastoma in children: conclusions from the Children's Cancer Group 921 randomized phase III study. J Clin Oncol 1999;17:832-45

16. Kühl J, Müller HL, Berthold F, et al. Pre-radiation chemotherapy of children and young adults with malignant brain tumors: results of German pilot trial HIT'88/89. Klin Paeditr 1998;17:832-45

17. Krischer JP, Ragap AH, Kun L, et al. Nitrogen mustard, vincristine, procarbazine and prednisone as adjuvan chemotherapy in the treatment of medulloblastoma. A Pediatric Oncology Group study. J Neurosurg 1991;74:905-9

18. Bailey CC, Gnekow A, Wellek S, et al. Prospective randomised trial of chemotherapy given before radiotherapy in childhood medulloblastoma. International Society of Paediatric Oncology (SIOP) and the (German) Society of Paediatric Oncology (GPO): SIOP II. Med Pediatr Oncol 1995;25:166-78

19. Evans AE, Jenkin RD, Sposto R, et al. The treatment of medulloblastoma. Results of a prospective randomized trial of radiation therapy with and without CCNU, vincristine, and prednisone. J Neurosurg 1990;72:572-82

20. Tait DM, Thornton-Jones H, Bloom HJ, Lemerle J, Morris JP. Adjuvant chemotherapy for medulloblastoma: the first multi-centre control trial of the International Society of Paediatric Oncology (SIOP I). Eur J Cancer 1990;26:464-9

21. Schell MJ, McHaney VA, Green AA, et al. Hearing loss in children and young adults receiving cisplatin with or without prior cranial irradiation. J Clin Oncol 1989;7:754-60

22. Ertaş G, Uçer AR, Altundag MB, Durmus S, Calikoglu T, Ozbagi K, Abanuz $\mathrm{H}$, Altundag K, Demirkasimoglu A. Medulloblastoma/primitive neuroectodermal tumor in adults: prognostic factors and treatment results: a single-center experience from Turkey. Med Oncol 2008;25:69-72

23. Thomas PR, Deutsch M, Kepner JL, et al. Low-stage medulloblastoma: final analysis of trial comparing standard-dose with reduced-dose neuraxis irradiation. J Clin Oncol 2000;18:3004-11

24. St. Clair WH, Adams JA, Bues M, et al. Advantage of protons compared to conventional X-Ray or IMRT in the treatment of a pediatric patient with medulloblastoma. Int J Radiat Oncol Biol Phys 2004;38:727-34

25. Jain N, Krull KR, Brouwers P, Chintagumpala MM, Woo SY. Neuropsychological outcome following intensity-modulated radiation therapy for pediatric medulloblastoma. Pediatr Blood Cancer 2008;51:2759 
26. Lin R, Hug EB, Schaefer BS, et al. Conformal proton radiation therapy of the posterior fossa: A study comparing protons with three-dimensional planned photons in limiting dose to auditory structures. Int $\mathbf{J}$ Radiat Oncol Biol Phys 2000;48:1219-26

27. Parker W, Filion E, Roberge D, Freeman CR. Intensity-modulated radiotherapy for craniospinal irradiation: target volume considerations, dose constraints, and competing risks Int J Radiat Oncol Biol Phys. 2007;69:251-7

28. Parker WA, Freeman CR. A simple technique for craniospinal radiotherapy in the supine position. Radiother Oncol 2006;78:217-22

29. Liu M, Carrie C, Parker W, Freeman CR. Comprasion of manual and computer-generated customized blocks for whole brain fields used in the treatment of medulloblastoma. Med Pediatr Oncol 2002;38:55-7
30. Carrie C, Hoffstetter S, Gomez F, et al. Impact of targeting deviations on outcome in medulloblastoma:Study of the French Society of Pediatric Oncology (SFOP).Int J Radiat Oncol Biol Phys 1999;45:435-9

31. Hall EJ, Wuu CS. Radiation-induced second cancers:The impact of 3D-CRT and IMRT. Int $\mathrm{J}$ Radiat Oncol Biol Phys 2003;56:83-8

32. International Commission on Radiation Units and Measurements. Prescribing, recording, and reporting photon beam therapy. Bethesda, MD:ICRU;1999

33. Survivors of Childhood Cancer: Assessment and Management. St. Louis, Mo: Mosby; 1994.

34. Brandes AA, Franceschi E, Tosoni A. Adult neuroectodermal tumors of posterior fossa (medulloblastoma) and of supratentorial sites (stPNET). Crit Rev Oncol Hematol 2009;71:165-79 